\section{CPS-322 ADALIMUMAB CONCENTRATIONS PRIOR TO THE IMPLEMENTATION OF THERAPEUTIC DRUG MONITORING IN PATIENTS WITH INFLAMMATORY BOWEL DISEASE}

A Gracia Moya*, A Pau Parra, P Sánchez Sancho, I Cardona Pascual, P Garcia Ortega, B Torrecilla Vall-Llossera, MQ Gorgas Torner, M Miarons Font. Vall D'hebron Hospital, Hospital Pharmacist, Barcelona, Spain

\subsection{6/ejhpharm-2021-eahpconf.154}

Background and importance Adalimumab (ADA), an anti-TNF agent, has been shown to effectively induce and maintain remission in patients with inflammatory bowel disease (IBD). The usual recommended dose in the maintenance phase is 40 mg every 14 days. The formation of anti-adalimumab antibodies (AAA) reduces plasma ADA serum concentrations (ADAsc), as well as its efficacy.

Aim and objectives The aim of the study was to analyse ADAsc and the presence of AAA in patients with IBD prior to the implementation of dose optimisation through therapeutic drug monitoring (TDM). The secondary objectives were to evaluate changes in the posology and to characterise the symptoms of IBD.

Material and methods A retrospective observational study was conducted in patients with Crohn's disease (CD) or ulcerative colitis (UC) on maintenance therapy with ADA during a follow-up period of 3 years (July 2016 to April 2019) before applying TDM in a tertiary referral centre. The concentrations of $\mathrm{ADA}$ and $\mathrm{AAA}$ were determined by enzyme linked immunosorbent assay. The therapeutic range (TR) of ADA, according to the hospital protocol, is $8-12 \mu \mathrm{g} / \mathrm{mL}$. Biodemographic, analyti$\mathrm{cal}$ and clinical data were collected from the clinical history.

Results 165 patients $(53.0 \%$ women $)$ were included, 132 $(80.0 \%)$ with CD. Mean age was 40.00 (SD 12.4) years. At the beginning of the study, $119(72.1 \%)$ patients received ADA $40 \mathrm{mg}$ every 14 days and 46 (27.1\%) intensified regimens. The average concentration of ADA was 7.24 (SD 3.6) $\mu \mathrm{g} / \mathrm{mL}$ and $82(49.7 \%)$ patients had ADAsc outside the TR. We observed AAA in $16(9.7 \%)$ patients. Of these, 15 (93.7\%) had ADAsc outside the TR. During the study, regimen intensification was conducted in 34 (20.6\%) patients. Finally, regarding patients with ADAsc outside the TR, 35 $(42.7 \%)$ presented with abdominal symptoms and/or systemic manifestations.

Conclusion and relevance ADAsc outside the TR were observed in half of the patients, in approximately 1 in every 10 patients AAA were detected and $42.7 \%$ of patients with ADAsc outside the TR presented with symptoms. Implementation of a TDM might be a useful tool for managing patients with IBD on biologic therapy to reduce the number of patients with ADAsc outside the TR.

\section{REFERENCES AND/OR ACKNOWLEDGEMENTS}

Conflict of interest No conflict of interest

\section{CPS-323 EVALUATION OF THE USE, ADHERENCE AND TOLERANCE OF $0.03 \%$ TACROLIMUS EYE DROPS}

I Varela Rey*, M Gonzalez-Barcia, L García-Quintanilla, A Castro-Balado, M Busto-Iglesias, I Zarra-Ferro, A Fernández-Ferreiro. Complejo Hospitalario Universitario De Santiago De Compostela, Hospital Pharmacist Service, Santiago De Compostela, Spain

10.1136/ejhpharm-2021-eahpconf.155
Background and importance Tacrolimus is an immunosuppressant with many potential uses in ophthalmic diseases. It is an inhibitor of calcineurin phosphatases, which suppress the first phase of $T$ cell activation and leads to a decrease in inflammatory activity. There are no commercialised eye drops in Spain so its formulation as a pharmaceutical compound has to be done by the hospital pharmacy services.

Aim and objectives To evaluate the use, tolerance and adherence of $0.03 \%$ tacrolimus eye drops as a pharmaceutical compound.

Material and methods A retrospective observational study was conducted in patients treated with $0.03 \%$ tacrolimus eye drops from January 2017 to March 2020. The eye drops were prepared and dispensed by the hospital pharmacy service. For the preparation, $0.6 \mathrm{~mL}$ of intravenous Prograf $5 \mathrm{mg} / \mathrm{mL}$ were diluted with Liquifilm to a final volume of $10 \mathrm{~mL}$.

Demographic (sex and age) and clinical data (diagnosis, duration, adherence and tolerance) were recorded using electronic prescription and electronic medical records. Adherence was measured using registered dispensations as well as by follow-up controls by an ophthalmologist. We classified as the most compliant patients those with no delay in dispensation times in the pharmacist consultation.

Results 54 patients $(57 \%$ men) with a mean age of $32 \pm 21$ years used tacrolimus eye drops during the study period. Tacrolimus eye drops were used for the treatment of immune mediated ophthalmic inflammatory diseases in $61.8 \%$ of patients, of whom $49.1 \%$ were for atopic or vernal keratoconjunctivitis. $20.0 \%$ of the total number of patients used the eye drops for hyperaemia of unknown cause, $12.7 \%$ were used for dry eye and the remaining $5.4 \%$ for the treatment of graft rejections.

Mean duration of treatment was $1.8 \pm 1.0$ years. Tolerance was generally good. Only $24.07 \%$ of patients presented with itching, and 2 patients $(3.70 \%)$ had palpebral dermatitis and miosis. $50 \%$ of patients were highly compliant (having no delay in dispensations), $38.89 \%$ had delayed dispensing at least once, 9.26\% had delayed dispensing at least twice and $1.85 \%$ had irregular dispensations.

Conclusion and relevance $0.03 \%$ tacrolimus eye drops were used primarily for the treatment of vernal or atopic keratoconjunctivitis but they have wide potential uses in ophthalmology diseases. The formulation was well tolerated by most of patients and adherence was generally correct, as measured by the pharmacist consultations.

\section{REFERENCES AND/OR ACKNOWLEDGEMENTS}

Conflict of interest No conflict of interest

\section{CPS-324 EFFECTIVENESS AND SAFETY OF IXEKIZUMAB IN MODERATE-TO-SEVERE PLAQUE PSORIASIS}

${ }^{1}$ MDP Briceño Casado*, ${ }^{2} \mathrm{MD}$ Gil-Sierra, ${ }^{1} \mathrm{~B}$ De La Calle Riaguas, ${ }^{3} \mathrm{M}$ Dominguez-Cantero. ${ }^{1}$ Hospital Nuestra Señora Del Prado, Hospital Pharmacy, Talavera De La Reina, Spain; ${ }^{2}$ Hospital Doctor Jose Molina Orosa, Hospital Pharmacy, Lanzarote, Spain; ${ }^{3}$ Hospital Universitario Puerto Real, Hospital Pharmacy, Cadiz, Spain

\subsection{6/ejhpharm-2021-eahpconf.156}

Background and importance Ixekizumab is a high affinity monoclonal antibody against interleukin 17A. It is used for the treatment of moderate-to-severe plaque psoriasis (MTSPP). 